\title{
Distributed Systems and Algorithms
}

\author{
Omer Rana ${ }^{1}$, Giandomenico Spezzano ${ }^{1}$, Michael Gerndt ${ }^{2}$, and Daniel S. Katz ${ }^{2}$ \\ 1 Topic Chairs \\ 2 Members
}

Despite an impressive body of research, parallel and distributed computing remains a complex task prone to subtle software issues that can affect both the correctness and the performance of the computation. The increasing demand to distribute computing over large-scale parallel and distributed platforms, such as grids and large clusters, often combined with the use of hardware accelerators, overlaps with an increasing pressure to make computing more dependable. To address these challenges, the parallel and distributed computing community continuously requires better tools and environments to design, program, debug, test, tune, and monitor parallel programs. This topic aims to bring together tool designers, developers, and users to share their concerns, ideas, solutions, and products covering a wide range of platforms, including homogeneous and heterogeneous multi-core architectures. Contributions with solid theoretical foundations and experimental validations on production-level parallel and distributed systems were particularly valued. This year, we encouraged submissions proposing intelligent monitoring and diagnosis tools and environments, which can exploit behavior knowledge to detect programming bugs or performance bottlenecks and help ensure correct and efficient parallel program execution.

This year nineteen papers were submitted to Topic 8 representing a wide spectrum of research areas in distributed computing. Of the nineteen submissions, seven have been accepted for publication. Each paper was reviewed by four reviewers (two papers received only three reviews).

This year 20 papers were submitted to this topic. Each paper was reviewed by at least three reviewers and we selected 7 papers as definite accepts for the conference $(35 \%)$. It was interesting to see papers focusing on emerging themes such as multi-core and GPUs, along with papers that covered more established themes such as program profiling, performance analysis, work ow management and application tuning. The paper entitled "Starscheck: A tool to find errors in task-based parallel programs" received the highest review score and was accepted as a distinguished paper. This paper discusses a tool to debug Star Superscalar programs, producing a warning if a task or the main thread within a program generates an invalid access.

We would like to thank the authors who submitted a contribution, the EuroPar Organizing Committee, and the referees who provided useful and timely comments. 